\title{
Dynamics of competitive interaction between two mining enterprises and one processing: existence and stability of the equilibrium point
}

\author{
Aleksandr Kirjanen ${ }^{1}$, Oleg Malafeyev ${ }^{1}$, Irina Zaitseva ${ }^{2,}{ }^{*}$, Aleksandr Kovshov ${ }^{1}$, and Dmitry \\ Kolesov ${ }^{1}$ \\ ${ }^{1}$ Saint-Petersburg State University, 7/9 Universitetskaya nab., Saint-Petersburg, 199034, Russia \\ ${ }^{2}$ Stavropol State Agrarian University, 12 Zootechnicheskiy Ln, Stavropol, Russia, 355017
}

\begin{abstract}
A mathematical model of dynamic interaction between two mining enterprises and one processing is formalized and studied in the paper. The process of interaction is described by a system of three delay differential equations. The criterion for asymptotic stability of nontrivial equilibrium point is obtained when all three enterprises co-work steadily. The problem is reduced to finding stability criterion for quasi-polynomial of third order. We found time intervals between deliveries of raw materials and manufacturing of finished products, in which the process of interaction of the three agents is stable.
\end{abstract}

\section{Introduction}

Let us consider the dynamics of competitive interaction between two mining enterprises and one processing, manufacturing finished products from the supplied raw materials. We will assume that we know the necessary amount of finished products for the region in which these products are sold. In this case, competition occurs between mining enterprises, which is characterized by the optimal distribution between them of the amount of extracted raw materials.

The mathematical model of this process is described by a system of three delay differential equations. These equations are determined so that the nontrivial equilibrium point of the system is, as described above, three optimal values. The task is: to find the conditions under which the solution of the system will tend to the optimal equilibrium point. Conditions will be expressed only through the coefficients of the original system.

These coefficients depend on the technology of extraction and manufacturing of finished products (technological coefficients), on the degree of competition between mining enterprises, on the value of supplies of raw materials to a processing company, on the time intervals in the supply of raw materials to the consumer and on the time on which finished products are manufactured. The task is to describe under which dependence and constraints on the coefficients the stability of the entire system is preserved.

\footnotetext{
* Corresponding author: irina.zaitseva.stv@yandex.ru
} 
A similar problem for two agents was considered in $[1 ; 2 ; 3 ; 4 ; 5]$ and it was described by a system of two nonlinear delay differential equations. In particular, for the model of competition between two investors, delay played the role of capital turnover time. Moreover, the system was described in such a way that the stable co-work of both investors depended primarily on the coefficients of mutual competition, or rather, on their product. In all articles, the search for coefficient criteria for the asymptotic stability of the equilibrium state was reduced to finding conditions under which the roots of the characteristic quasipolynomial of the linearized system lay in the left half-plane. In [6; 7], coefficient criteria under which a system characterizing the dynamics between the extractive and processing industries is stable are indicated. In [8] the influence of the capital turnover time on the stability of the equilibrium point of two competing firms is obtained.

Also in the article the maximum admissible time interval in both the supply of raw materials and the sale of finished products, called the maximum admissible delay, which will maintain a balance between production and processing is obtained.

\section{Mathematical model with two mining and one processing enterprises}

We will assume that the necessary and economically justified quantity of finished product $K$ is known for this region. Then, according to the technology, the first two enterprises need to extract raw materials in the amount of $K_{1}$ and $K_{2}$ respectively. Let's write down a mathematical model of the process of mutual influence of three agents in such a way that its nontrivial equilibrium position is a point $\left(K_{1}, K_{2}, K\right)$ :

$$
\begin{gathered}
\frac{d P_{1}(t)}{d t}=r_{1}\left(1-\frac{P_{1}\left(t-h_{1}\right)}{K_{1}}+\beta_{1}\left(1-\frac{P_{2}(t)}{K_{2}}\right)+\gamma_{1}\left(1-\frac{Q(t)}{K}\right)\right) P_{1}(t) \\
\frac{d P_{2}(t)}{d t}=r_{2}\left(1-\frac{P_{2}\left(t-h_{2}\right)}{K_{2}}+\beta_{2}\left(1-\frac{P_{1}(t)}{K_{1}}\right)+\gamma_{2}\left(1-\frac{Q(t)}{K}\right)\right) P_{2}(t) \\
\frac{d Q(t)}{d t}=\left(-c+l_{1} \frac{P_{1}(t)}{K_{1}}+l_{2} \frac{P_{2}(t)}{K_{2}}-l \frac{Q\left(t-h_{3}\right)}{K}\right) Q(t)
\end{gathered}
$$

In this system, $P_{1}, P_{2}$ are the raw materials produced by the first and second enterprises, respectively, and $\mathrm{Q}$ - the quantity of the finished product produced by the third enterprise. $r_{1}, r_{2}$ - technological coefficients that characterize the production of raw materials by the first two enterprises; $\beta_{1}, \beta_{2}$ - competition coefficients that characterize the degree of pressure of one mining enterprise on another. Coefficients $\gamma_{1}, \gamma_{2}$ characterize the share of raw materials coming from each of the suppliers to the processing enterprise; $h_{1}, h_{2}$ - time intervals through which raw materials are sent to the processing enterprise.

Coefficient $c>0$ - the rate of reduction of finished production in the absence of raw materials (in the system is marked with a minus sign). The coefficient $l$ characterizes the share of old stocks of finished products in the processing enterprise, and the coefficients $l_{1}, l_{2}$ characterize the share of raw materials consumed by the third enterprise; delay $h_{3}$ is the time of sale of the finished product. 
If the condition $l_{1}+l_{2}=l+c$ is met, the nontrivial equilibrium position of the system (1) is a point $\left(K_{1}, K_{2}, K\right)$. We'll find it conditions under which this equilibrium point is asymptotically stable.

\section{Linearized system and characteristic equation. Asymptotic stability of a nontrivial equilibrium point at zero delays}

To study the stability of the non-trivial equilibrium point of the system (1), we move from the equilibrium point $\left(K_{1}, K_{2}, K\right)$ to the equilibrium position $(0,0,0)$, making the substitution of variables

$$
P_{j}(t)=K_{j}\left(1+x_{j}(t)\right), j=1,2 ; \quad Q(t)=K(1+z(t)) .
$$

For the new system, we write only its linear part, discarding the quadratic terms, which, according to the theory of differential equations, will not affect the asymptotic stability of the trivial equilibrium point:

$$
\begin{gathered}
\frac{d x_{1}(t)}{d t}+r_{1}\left(x_{1}\left(t-h_{1}\right)+\beta_{1} x_{2}(t)+\gamma_{1} z(t)=0\right. \\
\frac{d x_{2}(t)}{d t}+r_{2}\left(x_{2}\left(t-h_{2}\right)+\beta_{2} x_{1}(t)+\gamma_{2} z(t)=0\right. \\
\frac{d z(t)}{d t}+\operatorname{lz}(t)+\operatorname{lz}\left(t-h_{3}\right)-l_{1} x_{1}(t)-l_{2} x_{2}(t)=0
\end{gathered}
$$

The characteristic equation corresponding to system (2) has the form:

$$
\left|\begin{array}{ccc}
\lambda+r_{1} e^{-\lambda h_{1}} & \beta_{1} r_{1} & \gamma_{1} r_{1} \\
\beta_{2} r_{2} & \lambda+r_{2} e^{-\lambda h_{2}} & \gamma_{2} r_{2} \\
-l_{1} & -l_{2} & \lambda+l+l e^{-\lambda h_{3}}
\end{array}\right|=0 .
$$

First, let's consider the case when all the delays $h_{1}=h_{2}=h_{3}=0$. After calculating the determinant, we get:

$$
\begin{aligned}
& \lambda^{3}+\left(r_{1}+r_{2}+2 l\right) \lambda^{2}+\left(r_{1} r_{2}+2 l\left(r_{1}+r_{2}\right)+l_{1} \gamma_{1}+l_{2} \gamma_{2}-\beta_{1} \beta_{2} r_{1} r_{2}\right) \lambda+ \\
& +2 l r_{1} r_{2}+l_{1} \gamma_{1} r_{2}+l_{2} \gamma_{2} r_{1}-l_{1} \beta_{1} \gamma_{2} r_{1}-l_{2} \beta_{2} \gamma_{1} r_{2}-2 l \beta_{1} \beta_{2} r_{1} r_{2}=0
\end{aligned}
$$

The trivial equilibrium point of the system (2) is asymptotically stable if and only if the roots of the characteristic equation (3) lie in the left half-plane. For zero delays, the roots of the polynomial (4) will be in the left half-plane if and only if the coefficients of the thirddegree polynomial are positive, i.e.

$$
\begin{gathered}
r_{1} r_{2}+2 l\left(r_{1}+r_{2}\right)+l_{1} \gamma_{1}+l_{2} \gamma_{2}>\beta_{1} \beta_{2} r_{1} r_{2} \\
2 l r_{1} r_{2}+l_{1} \gamma_{1} r_{2}+l_{2} \gamma_{2} r_{1}>l_{1} \beta_{1} \gamma_{2} r_{1}+l_{2} \beta_{2} \gamma_{1} r_{2}+l \beta_{1} \beta_{2} r_{1} r_{2} .
\end{gathered}
$$

Inequality (5) means that the mutual competition between two extractive enterprises should not be large and the product of the coefficients characterizing competition is limited by the upper-left part of the inequality (5). Inequality (6) also gives restrictions on the coefficients of competition $\beta_{1}$ and $\beta_{2}$. 
If we consider a particular case of competition between two mining companies in the absence of a third processing, then all the coefficients $l=l_{1}=l_{2}=0$. In this case, from inequality (5) we have

$$
r_{1} r_{2}>\beta_{1} \beta_{2} r_{1} r_{2} \text { or } \beta_{1} \beta_{2}<1 \text {. }
$$

Inequality (7) is a necessary and sufficient condition for the stable coexistence of two competitors, two investors, two competing for a common food of individuals and is found in the works $[1 ; 2 ; 3 ; 4 ; 5]$. Note the following fact. Biologists divide the coefficients of a biological system into primary and secondary characteristics. The primary characteristics are coefficients that characterize the natural growth of the population, and the secondary ones are competition coefficients. In biological studies and observations, it is indicated that the stability of the biosystem is primarily affected by secondary features. Inequality (7) indicates that the stability of an ecosystem or biosystem is primarily affected by the interaction of secondary features, which should be small.

\section{Quasi-polinomial and its roots with identical delays}

Consider the characteristic equation (3) for identical delays $h_{1}=h_{2}=h_{3}=h>0$. Let's calculate the determinant (3), writing it in descending order by degrees $\lambda$ and ascending by degrees $e^{-\lambda h}$ :

$$
\begin{aligned}
& \Delta=\lambda^{3}+l \lambda^{2}+\left(r_{1}+r_{2}+l\right) \lambda^{2} e^{-\lambda h}+\left(l_{1} \gamma_{1} r_{1}+l_{2} \gamma_{2} r_{2}-\beta_{1} \beta_{2} r_{1} r_{2}\right) \lambda+l\left(r_{1}+r_{2}\right) \lambda e^{-\lambda h}- \\
& -\left(l_{1} \beta_{1} \gamma_{2}+l_{2} \beta_{2} \gamma_{1}+l \beta_{1} \beta_{2}\right) r_{1} r_{2}+\left(l_{1} \gamma_{1}+l_{2} \gamma_{2}-l \beta_{1} \beta_{2}\right) r_{1} r_{2} e^{-\lambda h}+l r_{1} r_{2} e^{-2 \lambda h}+l r_{1} r_{2} e^{-3 \lambda h}=0
\end{aligned}
$$

Multiplying equality (8) by $h^{3} e^{3 \lambda h}$ and making a change of variable in the resulting equation $z=h \lambda$, we get that the third degree quasi-polynomial equals to zero with respect to $\mathrm{z}$ and $e^{z}$ :

$$
\begin{aligned}
& H\left(z, e^{z}\right)=z^{3} e^{3 z}+l h z^{2} e^{3 z}+\left(r_{1}+r_{2}+l\right) h z^{2} e^{2 z}+\left(l_{1} \gamma_{1} r_{1}+l_{2} \gamma_{2} r_{2}-\beta_{1} \beta_{2} r_{1} r_{2}\right) h^{2} z e^{3 z}+ \\
& +l\left(r_{1}+r_{2}\right) h^{2} z e^{2 z}-\left(l_{1} \beta_{1} \gamma_{2}+l_{2} \beta_{2} \gamma_{1}+l \beta_{1} \beta_{2}\right) r_{1} r_{2} h^{3} e^{3 z}+\left(l_{1} \gamma_{1}+l_{2} \gamma_{2}-l \beta_{1} \beta_{2}\right) r_{1} r_{2} h^{3} e^{2 z}+ \\
& +l r_{1} r_{2} h^{3} e^{z}+l r_{1} r_{2} h^{3}=0
\end{aligned}
$$

Rearrange the terms in (9) in descending order of exponential powers multiplied by the corresponding polynomial:

$$
\begin{aligned}
& H(z)=\left(z^{3}+l h z^{2}+\left(l_{1} \gamma_{1} r_{1}+l_{2} \gamma_{2} r_{2}-\beta_{1} \beta_{2} r_{1} r_{2}\right) h^{2} z-\left(l_{1} \beta_{1} \gamma_{2}+l_{2} \beta_{2} \gamma_{1}+l \beta_{1} \beta_{2}\right) r_{1} r_{2} h^{3}\right) e^{3 z}+ \\
& +\left(\left(r_{1}+r_{2}+l\right) h z^{2}+l\left(r_{1}+r_{2}\right) h^{2} z+\left(l_{1} \gamma_{1}+l_{2} \gamma_{2}-l \beta_{1} \beta_{2}\right) r_{1} r_{2} h^{3}\right) e^{2 z}+l r_{1} r_{2} h^{3} e^{z}+l r_{1} r_{2} h^{3}=0
\end{aligned}
$$

To shorten the entry, we denote

$$
\begin{aligned}
& p_{1}=l h, p_{2}=\left(l_{1} \gamma_{1} r_{1}+l_{2} \gamma_{2} r_{2}-\beta_{1} \beta_{2} r_{1} r_{2}\right) h^{2}, p_{3}=\left(l_{1} \beta_{1} \gamma_{2}+l_{2} \beta_{2} \gamma_{1}+l \beta_{1} \beta_{2}\right) r_{1} r_{2} h^{3}, \\
& q_{1}=\left(r_{1}+r_{2}+l\right) h, q_{2}=l\left(r_{1}+r_{2}\right) h^{2}, q_{3}=\left(l_{1} \gamma_{1}+l_{2} \gamma_{2}-l \beta_{1} \beta_{2}\right) r_{1} r_{2} h^{3}, u=l r_{1} r_{2} h^{3}, \\
& \mathrm{v}=l r_{1} r_{2} h^{3}
\end{aligned}
$$

Then quasi-polinomial will look like:

$$
H(z)=\left(z^{3}+p_{1} z^{2}+p_{2} z-p_{3}\right) e^{3 z}+\left(q_{1} z^{2}+q_{2} z+q_{3}\right) e^{2 z}+u e^{z}+v=0 .
$$


It follows from the theorem of L. S. Pontryagin [9] that if a quasi-polinomial does not have a principal term, then there is a chain of its roots whose real parts tend to $+\infty$. In our case, the quasi-polinomial (12) has the principal term of the bi-degree $(3,3)$. We will look for conditions under which its roots lie in the left half-plane.

Substitute $\mathrm{z}=$ iy in quasi-polinomial (12) and select the real

$$
F(y)=\left(y^{3}-p_{2} y\right) \sin 3 y-\left(p_{1} y^{2}+p_{3}\right) \cos 3 y-\left(q_{1} y^{2}-q_{3}\right) \cos 2 y-q_{2} y \sin 2 y+u \cos y+v
$$

and imaginary parts

$$
G(y)=-\left(y^{3}-p_{2} y\right) \cos 3 y-\left(p_{1} y^{2}+p_{3}\right) \sin 3 y-\left(q_{1} y^{2}-q_{3}\right) \sin 2 y+q_{2} y \cos 2 y+u \sin y
$$

We will also need derivatives

$$
\begin{aligned}
& F^{\prime}(y)=\left(3 y^{2}-p_{2}\right) \sin 3 y+3\left(y^{3}-p_{2} y\right) \cos 3 y-2 p_{1} y \cos 3 y+3\left(p_{1} y^{2}+p_{3}\right) \sin 3 y-, \\
& -2 q_{1} y \cos 2 y+\left(2 q_{1} y^{2}-2 q_{3}\right) \sin 2 y-q_{2} \sin 2 y-2 q_{2} y \cos 2 y-u \sin y \\
& G^{\prime}(y)=\left(-3 y^{2}+p_{2}\right) \cos 3 y+3\left(y^{3}-p_{2} y\right) \sin 3 y-2 p_{1} y \sin 3 y-3\left(p_{1} y^{2}+p_{3}\right) \cos 3 y- \\
& -2 q_{1} y \cos 2 y+2\left(q_{1} y^{2}-q_{3}\right) \sin 2 y+q_{2} \cos 2 y-2 q_{2} y \sin 2 y+u \cos y
\end{aligned}
$$

Theorem 1. [4, p.78; 9]. In order for the roots of the quasi polinomial (12) lay in the left half-plane, it is necessary and sufficient that the vector of the amplitude-phase characteristic turns monotonously around the origin in a positive direction and with a positive speed, without passing through the origin. Analytically this is expressed by the following condition

$$
G^{\prime}(y) F(y)-F^{\prime}(y) G(y)>0 \text { for all } y .
$$

Remark 1. Inequality (17) means that the amplitude-phase the characteristic when it rotates around the origin intersects any straight line passing through a point $(0,0)$ at a positive angle and does not touch it.

According to the Pontryagin criterion [4; 9], the roots of the quasi-polinomial will be in the left half-plane if and only if the vector $w=H(i y)$ rotates around the origin by an angle $4 \pi k s+\pi r$, when $y \in[-2 \pi k ; 2 \pi k]$ for large $k$. that is, intersect the axes $F(y)$ and $G(y)$ $4 k s+r$ times. In our case, the quasi-polinomial (12) has a bi-degree (s=3, $r=3$ ), therefore, on the segment $[-2 \pi k ; 2 \pi k]$ functions $F(y)$ and $G(y)$ must have $12 k+3$ roots. With the exception of the segment $[-2 \pi ; 2 \pi]$ on the other segments of length $4 \pi$, functions $F(y)$ and $G(y)$ must have 12 roots.

The inequality (17), which characterizes a monotonous turn of the amplitude-phase characteristic in the positive direction at a point $y_{0}=0$, looks like this:

$$
G^{\prime}(0) F(0)=\left(p_{2}-3 p_{3}+q_{2}+u\right)\left(-p_{3}+q_{3}+u+v\right)>0
$$

In the problem under consideration (the smallness of the coefficients of mutual competition), inequality (18) is equivalent to a system of inequalities

$$
\begin{gathered}
q_{3}+u+v>p_{3} \\
p_{2}+q_{2}+u>3 p_{3} .
\end{gathered}
$$

Returning to the coefficients of the system (2), from (19) we obtain 


$$
\begin{gathered}
l_{1} \gamma_{1}+l_{2} \gamma_{2}+2 l>l_{1} \gamma_{2} \beta_{1}+l_{2} \gamma_{1} \beta_{2}+2 l \beta_{1} \beta_{2} \\
l_{1} \gamma_{1} r_{1}+l_{2} \gamma_{2} r_{2}+l\left(r_{1}+r_{2}+r_{1} r_{2}\right)>3\left(l_{1} \gamma_{2} \beta_{1}+l_{2} \gamma_{1} \beta_{2}+l \beta_{1} \beta_{2}\right) r_{1} r_{2} h+\beta_{1} \beta_{2} r_{1} r_{2} .
\end{gathered}
$$

The first inequality in (20) is always satisfied if the competition between extractive enterprises is small ( $\beta_{1} \beta_{2}<1$ ), which is consistent with the above statements about the stability of economic and biological systems. The second inequality in (20) imposes stricter restrictions on the competition coefficients. Moreover, from the second inequality we obtain the maximum admissible delay $h_{\max }$, in which there will be no loss of stability of the economic system:

$$
h_{\max }=\frac{l_{1} \gamma_{1} r_{1}+l_{2} \gamma_{2} r_{2}+l\left(r_{1}+r_{2}+r_{1} r_{2}\right)-\beta_{1} \beta_{2} r_{1} r_{2}}{3\left(l_{1} \gamma_{2} \beta_{1}+l_{2} \gamma_{1} \beta_{2}+l \beta_{1} \beta_{2}\right) r_{1} r_{2}} .
$$

Now we give a stability criterion for the quasi-polynomial (12) with non-negative coefficients. In contrast to the Hermite - Biller criterion we will require that inequality (17) be satisfied not at the roots of the functions $F(y)$ or $G(y)$, but at the points of their intersection.

Theorem 2. The roots of a quasi-polynomial (12) with non-negative coefficients lie in the left half-plane if the following conditions are fulfilled:

1. $G(0)=0, G^{\prime}(0)>0, F(0)>0, F^{\prime}(0)=0, F^{\prime}(+0)<0, F^{\prime}(-0)>0$

2. The equation $F(y)=G(y)$ has 6 roots on the segment $[0 ; 2 \pi]$, and

$$
y_{1} \in\left(0 ; \frac{\pi}{3}\right), y_{2} \in\left(\frac{\pi}{3} ; \frac{2 \pi}{3}\right), \ldots, y_{6} \in\left(\frac{5 \pi}{3} ; 2 \pi\right) ;
$$

3. At the points $y_{i}$, the graphs of the functions $F(y)$ and $G(y)$ intersect and the inequalities hold at these points:

$$
\begin{aligned}
& \text { if } y_{1} \in\left(0 ; \frac{\pi}{3}\right), G\left(y_{1}\right)=F\left(y_{1}\right)>0, G^{\prime}\left(y_{1}\right)>0, F^{\prime}\left(y_{1}\right)<0, \\
& \text { if } y_{2} \in\left(\frac{\pi}{3} ; \frac{2 \pi}{3}\right) G\left(y_{2}\right)=F\left(y_{2}\right)<0, G^{\prime}\left(y_{2}\right)<0, F^{\prime}\left(y_{2}\right)>0 \\
& \text { if } y_{6} \in\left(\frac{5 \pi}{3} ; 2 \pi\right) G\left(y_{6}\right)=F\left(y_{6}\right)<0, G^{\prime}\left(y_{6}\right)<0, F^{\prime}\left(y_{6}\right)>0
\end{aligned}
$$

or similar conditions are fulfilled with the replacement of inequalities by opposite.

Proof. Necessity. If all the roots of the quasi-polynomial (12) lie in the left half-plane, then with increasing $y$ the amplitude-phase characteristic monotonously rotates around the origin in the positive direction without passing through the point $(0 ; 0)$. This means that inequality (17) holds for all y. Therefore, all inequalities in formulas (22) - (24) are satisfied.

Sufficiency. It follows from formulas (13) - (16) that the function $G(y)$ is odd, and its derivative is even. The first part of condition 1 means that the function $G(y)$ increases passing through the point $(0 ; 0)$. On the other hand, the function $F(y)$ is even, but its derivative is odd and it follows from conditions 1 that at the point $y_{0}=0$ the function 
$F(y)$ has a positive maximum. Then the function $F(y)$ will decrease, passing the point $y_{0}=0$, while the function $G(y)$ in the same interval will increase.

Let $y_{1}$ be the intersection point of these graphs. At this point, the functions $F(y)$ and $G(y)$ are positive, with $G(y)$ increasing and $F(y)$ decreasing. Therefore, at the point, the first of inequalities (24) holds. With further movement, the function $F(y)$ intersects the axis $O y$ and at this point $F^{\prime}(y)<0$, a $G(y)>0$, as well. Therefore, inequality (17) also holds. The sign of the derivative $G^{\prime}(y)$ at this point is unimportant, since $F(y)=0$ in it.

According to the properties of trigonometric polynomials, the function $G(y)$ reaches its positive maximum before the function $F(y)$ reaches a negative minimum. After the maximum, the function $G(y)$ decreases, and after the minimum, the function $F(y)$ increases. Their new intersection at the point $y_{2}$ will occur at negative values of $F(y)$ and $G(y)$. This means that before the point $y_{2}$ there will be a root of the function $G(y)$.

Thus, at the point $y_{0}=0$ was the root of the function $G(y)$, then the root of $F(y)$, and then again the root of the function $G(y)$. We get the alternation of the roots of the functions $G(y)$ and $F(y)$ and the alternation of their intersection points, and the intersections occur in the intervals (23). From all this it follows that the amplitude-phase characteristic rotates monotonically around the origin and the roots of the quasi-polynomial lie in the left half-plane. C.T.D.

Remark 2. If conditions 1 and 2 of Theorem 2 are fulfilled, then it is sufficient to verify conditions 3 only at the point $y_{1} \in\left(0 ; \frac{\pi}{3}\right)$. All other chains of inequalities (24) will be automatically fulfilled.

It follows from the fact that the functions $F(y)$ and $G(y)$ are trigonometric polynomials of the same bi-degree; therefore, their roots and their intersection points have a periodicity of following each other. Inequalities (22) and (24) correspond to inequality (17). Whence it follows that the amplitude-phase characteristic will rotate monotonously around the origin. Therefore, all the roots of the quasi-polynomial (12) lie in the left half-plane.

Taking into account Remark 2, we now express the conditions of Theorem 2 in terms of the coefficients of the quasi-polynomial (12).

Theorem 3. The roots of a quasi-polynomial (12) lie in the left half-plane if and only if the following conditions are satisfied:

1.

$$
\begin{aligned}
& q_{3}+u+v>p_{3} \\
& p_{2}+q_{2}+u>3 p_{3} \\
& 2 p_{1}+2 q_{1}+6 p_{2}+4 q_{2}+4 q_{3}+u>9 p_{3}
\end{aligned}
$$

2. The equation

$$
\begin{aligned}
& y^{3}(\sin 3 y-\cos 3 y)-p_{1} y^{2}(\sin 3 y+\cos 3 y)-p_{2} y(\sin 3 y-\cos 3 y)-p_{3}(\sin 3 y+\cos 3 y)- \\
& -q_{1} y^{2}(\sin 2 y+\cos 2 y)-q_{2} y(\sin 2 y-\cos 2 y)+q_{3}(\sin 2 y+\cos 2 y)+u(\sin y+\cos y)+ \\
& +v=0
\end{aligned}
$$

has 6 roots on the segment $[0 ; 2 \pi]$, moreover 


$$
y_{1} \in\left(0 ; \frac{\pi}{3}\right), y_{2} \in\left(\frac{\pi}{3} ; \frac{2 \pi}{3}\right), \ldots, y_{6} \in\left(\frac{5 \pi}{3} ; 2 \pi\right)
$$

3.In the root $y_{1}$ of equation (26), the following inequalities hold:

$$
\begin{aligned}
& G\left(y_{1}\right)=-\left(y_{1}^{3}-p_{2} y_{1}\right) \cos 3 y_{1}-\left(p_{1} y_{1}^{2}+p_{3}\right) \sin 3 y_{1}-\left(q_{1} y_{1}^{2}-q_{3}\right) \sin 2 y_{1}+q_{2} y_{1} \cos 2 y_{1}+ \\
& +u \sin y_{1}>0 \\
& G^{\prime}\left(y_{1}\right)=\left(-3 y_{1}^{2}+p_{2}\right) \cos 3 y_{1}+3\left(y_{1}^{3}-p_{2} y_{1}\right) \sin 3 y_{1}-2 p_{1} y_{1} \sin 3 y_{1}-3\left(p_{1} y_{1}^{2}+p_{3}\right) \cos 3 y_{1}- \\
& -2 q_{1} y_{1} \cos 2 y_{1}+2\left(q_{1} y_{1}^{2}-q_{3}\right) \sin 2 y_{1}+q_{2} \cos 2 y_{1}-2 q_{2} y_{1} \sin 2 y_{1}+u \cos y_{1}>0 \\
& F\left(y_{1}\right)=\left(y_{1}^{3}-p_{2} y_{1}\right) \sin 3 y_{1}-\left(p_{1} y_{1}^{2}+p_{3}\right) \cos 3 y_{1}-\left(q_{1} y_{1}^{2}-q_{3}\right) \cos 2 y_{1}-q_{2} y_{1} \sin 2 y_{1}+ \\
& +u \cos y_{1}+v>0 \\
& F^{\prime}\left(y_{1}\right)=\left(3 y_{1}^{2}-p_{2}\right) \sin 3 y_{1}+3\left(y_{1}^{3}-p_{2} y_{1}\right) \cos 3 y_{1}-2 p_{1} y_{1} \cos 3 y_{1}+3\left(p_{1} y_{1}^{2}+p_{3}\right) \sin 3 y_{1}- \\
& -2 q_{1} y_{1} \cos 2 y_{1}+\left(2 q_{1} y_{1}^{2}-2 q_{3}\right) \sin 2 y_{1}-q_{2} \sin 2 y_{1}-2 q_{2} y_{1} \cos 2 y_{1}-u \sin y_{1}<0
\end{aligned}
$$

Proof. The first inequality in (25) corresponds to the condition $F(0)>0$ the second one to the condition $G^{\prime}(0)>0$, and the third inequality to the condition $F^{\prime}(0)<0$. The function $F(y)$ must decrease after the point 0 so its derivative must be negative. The derivative $F^{\prime}(y)$ is odd. From (15) we write out the leading term of the derivative for y in the first power:

$$
F^{\prime}(y) \approx\left(-3 p_{2}-3 p_{2}-2 p_{1}+9 p_{3}-2 q_{1}-4 q_{3}-2 q_{2}-2 q_{2}-u\right) y<0 \text { if } y>0 .
$$

From (31) we obtain one more condition on the coefficients of the quasi-polynomial (12) for which its roots lie in the left half-plane:

$$
2 p_{1}+2 q_{1}+6 p_{2}+4 q_{2}+4 q_{3}+u>9 p_{3} .
$$

Condition 2 of Theorem 3 is equivalent to condition 2 of Theorem 2. Condition 3 of Theorem 3 is equivalent to the first of conditions 3 of Theorem 2. In view of Remark 2, all conditions 3 of Theorem 2 are satisfied. Consequently, the amplitude-phase characteristic with an increase in y rotates monotonically around the origin in the positive direction and with a positive speed. This means that all the roots of the characteristic quasi-polynomial lie in the left half-plane.

If, using formulas (11), we express the conditions of Theorem 3 in terms of the coefficients of the original system (1), we obtain a criterion for the asymptotic stability of its nontrivial equilibrium point.

\section{Conclusions}

1. In this article the mathematical model of the dynamics of competitive interaction between two mining enterprises and one processing enterprise is formalized and studied.

2. In the particular case of system (1) in the absence of the third equation and zero delay, we obtain the condition $\beta_{1} \beta_{2}<1$. This inequality means that the stable coexistence of two competitors primarily depends on weak mutual competition [1-5].

3. In the work of L. S. Pontryagin [13] an asymptotic formula for the angle of rotation of the amplitude-phase characteristic is given. From this formula conditions when the roots of the quasi-polynomial lie in the left half-plane are obtained. Theorem 1 proved that the 
roots of a quasi-polynomial lie in the left half-plane if and only if the amplitude-phase characteristic rotates monotonically around the origin in a positive direction and with a positive speed. This result is valid not only for quasi-polynomials, but also for functions of a complex variable of a different kind.

4. In contrast to the Hermite - Biller conditions, which verify that inequality (17) is satisfied at the roots of the functions F (y) or G (y), in Theorem 2 it is proposed to check inequality (17) at the intersection points of the graphs of these functions, more precisely, only in one point.

5. Theorem 3 gives coefficient criteria for the stability of a third-order quasi-polynomial with respect to $\mathrm{z}$ and the exponent. Based on these criteria, it is indicated how to obtain the necessary and sufficient conditions for the stable coexistence of the economic system (1). (The conditions are long and the scope of this article does not fit).

6. The formula (21) shows the maximum admissible delay, in which there will be no loss of stability of the economic system.

The work is partially supported by the RFBR grant \# 18-01-00796.

\section{References}

1. Kiryanen A.I. The mathematical model of competition between two investors, taking into account the time of capital turnover // State and business. Questions of theory and practice: modeling, management, finance: Materials III Intern. - SPb .: Publ. SZAGS. 2011. p. 95-100.

2. Kirjanen A. Coefficient criteria of stable coexistence of two competitors, Stability and Control Processes 2015 - Proceedings. 2015. pp.463-466.

3. Hsu S.B. Predator - mediated coexistence and extinction. Biosc., Vol. 54, no. 3/4. 1981. pp. 231-248.

4. Kiryanen A.I. Veselov V.O. The effect of capital turnover time and coefficient criteria for the asymptotic stability of the equilibrium position of two competing firms // The State and Business. Issues of theory and practice: Proceedings of the V International Conference, North-West Institute of Management. - SPb .: CPI SZIU - phil. RANEPA, 2013. Pp. 50-62.

5. Kiryanen A.I. Stability of systems with aftereffect and their applications. St. Petersburg: Publishing House of St. Petersburg State University, 1994. 240 p.

6. Kostyukov K. I., Zaitseva I.V., Bondarenko G. V., Svechinskaya T. A. and Nechayeva S. V. Workforce planning as an element of control system Research journal of pharmaceutical biological and chemical sciences. 2016. Vol 7. no 6.

7. Zaitseva I. V., Kolesin I. D., Malafeyev O. A., Ermakova A. N. and Shlaev D. V. Modeling of the labour force redistribution in investment projects with account of their delay IEEE International Conference on Power, Control, Signals and Instrumentation Engineering. 2017.

8. Zaitseva I, Malafeyev O, Dolgopolova A, Zhukova V and Vorokhobina Y Numerical method for computing equilibria in economic system models with labor force AIP Conference Proceedings. 2116 450060. 2019.

9. Kirjanen A.I. Coefficient criteria of stable coexistence of two competitors // In 2015 International Conference "Stability and Control Processes" in Memory of V.I. Zubov (SCP). IEEE Xplore Digital Library. pp. 463-476.

10. Kirjanen A.I., Malafeyev O.A., Redinskikh N.D. Developing industries in cooperative interaction: equilibrium and stability in processes with lag // STATISTICS, OPTIMIZATION AND INFORMATION COMPUTING Stat., Optim. Inf. Comput., 
Vol. 5. 2017. pp 341-347.

11. Malafeyev O.A., Redinskikh N.D., Kirjanen A.I. Stability in the process with lag of interaction between mining and processing industries // Mathematics, Optimization and Control https:/e.mail.ru/messages/inbox/ 2018. arXiv.org > math > arXiv:1706.06010v1.

12. Kirjanen A.I., Logosha E.S. Influence of capital turnover time on the stability of equilibrium point of two competing firms // The XLVIII annual international conference on Control Processes and Stability (CPS'17). SPg: Publishing House Fedorova G.V., 2017. P.125.

13. Pontryagin L.S. On the zeros of some transcendental functions // Izv. USSR Academy of Sciences. Ser. mate. 1942.V.6. No. 3. Pp. 115-134. 